\title{
Remotely Sensed Ground Control Points
}

\author{
P. Hummel \\ CompassData, Inc, Centennial, Colorado, United States of America - phummel@compassdatainc.com
}

KEY WORDS: Aviation, Mapping, Aerodrome Mapping Database, AMDB, Satellite Imagery, Ground Truth, Feature Extraction, Do200A, Certified Dataset.

\begin{abstract}
:
Accurate ground control is required to georeferenced airborne and spaceborne images. The production of ortho-photogrammetric data requires ground control that is traditionally provided as Ground Control Points (GCPs) by GNSS measurements in the field. However, it can be difficult to acquire accurate ground control points due to required turn-around time, high costs or impossible access. CompassData, Inc. a specialist in ground control, has expanded its service to deliver Remotely Sensed Ground Control Points (RSGCPs®). TerraSAR-X and TanDEM-X are two satellites with such high accuracy of their orbital positions and SAR data that RSGCPs ${ }^{\circledR}$ can be produced to a sub-meter quality depending on certain parameters and circumstances. The technology and required parameters are discussed in this paper as well as the resulting accuracies.
\end{abstract}

\section{INTRODUCTION}

The data collected by the Airbus Space and Defence satellite TerraSAR-X allows to determine an accurate location of single features on the surface of the earth. A combination of multiple off-nadir viewing angles at a feature sets up an overdetermined solution for its position. Each of the viewing angles provides a SAR range and Doppler equation and satellite position based on highly accurate orbits. The so-called science orbit is in an accuracy range of several centimeters and the precise radar beam tracing and data resolution exceeds half a meter accuracy for feature location in the different modes in range and azimuth. The different modes are StripMap, SpotLight and Staring SpotLight with the last one being the most precise mode added in the end of 2013.

As in traditional photogrammetric stereo methodology, the processing of TerraSAR-X data introduces, per scene, two equations for each individual point measurement. Consequently, one pair provides four equations to determine a coordinate triplet (Easting, Northing, Elevation). To boost accuracy and confidence, multiple scenes (usually four) with different lookangles are used. The full performance range for viewing angles in SpotLight mode is between 20 and 55 degrees off-nadir.

A limiting factor for the highest possible theoretical accuracy is the availability of perfect features. Features considered perfect must fulfil both visibility requirements equally:

- Visibility in the SAR data

- Visibility in the optical image

Nevertheless, there have been massive scientific advances in this SAR technology, giving it new advantages in weatherindependency. Now, wherever ground surveying is not practical - or is simply impossible - this satellite program enables a remote collection of reliable ground control points. The RSGCPs ${ }^{\circledR}$ have an extremely high accuracy of under 1 meter and can be the anchors for improving traditional satellite imagery and/or airborne photos. The following article discusses the requirements of the features and the achieved accuracies of a field test.

\section{RSGCP® EXTRACTION METHOD}

The extraction of RSGCPs ${ }^{\circledR}$ requires multiple look angles. The figure below shows four look angles with two opposite-site dispositions and two same-side dispositions.

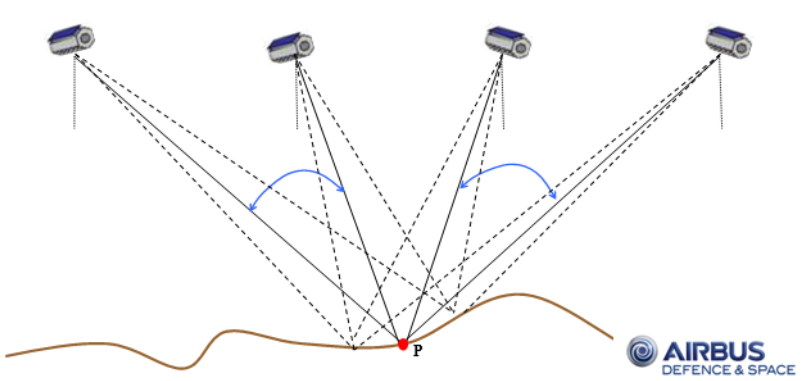

Figure 1. Satellite geometry for RSGCPs®.

Similar to other photogrammetric practises opposite-side stereo dispositions are preferred. These are achieved by a data acquisition of one ascending and one descending pass. Ideal intersection conditions are at 90 degrees for an opposite-side stereo constellation. As already mentioned more than two acquisitions are required; so more than one intersection angles occur resulting in a more robust solution for the coordinates of point $P$.

The increased over-determination of the equation system (SAR range and Doppler equations per view) helps to eliminate erroneous and unreliable points. This should be pointed out, because the choice of typical features is not as intuitive in the SAR data as it is in an optical dataset.

In a typical data sample, a traffic pole or street light are depicted as a bright spot for the high reflectivity.

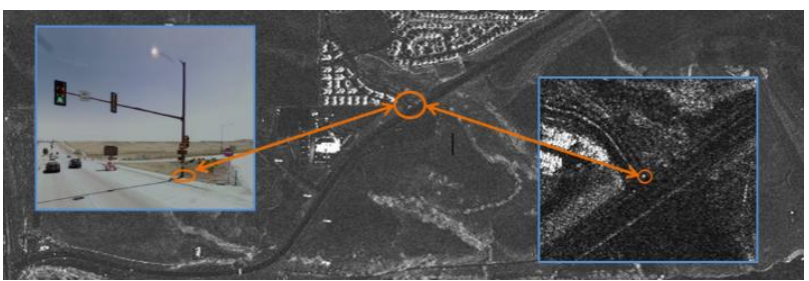

Figure 2. Location of a metal pole in the SAR data 
The active satellite sensor broadcasts signals, which are reflected by the ground. In case of a metal pole the signals are combined to a stronger reflection. Since these poles are standing vertically the effect adds up for the different ascending and descending passes of the TerraSar-X.
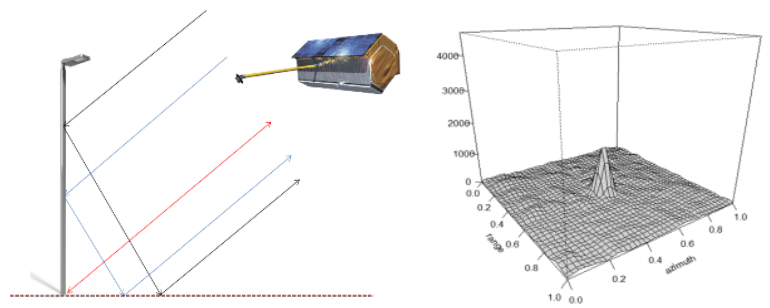

Figure 3. Metal pole and combination of reflections

\subsection{Features in the optical imagery}

The remotely sensed feature has to be clearly visible in the optical imagery to inherit the accurate geo reference without significant quality loss. In figure 4 are two examples of Pleiades imagery. While in the left image the large light pole and shadow create a clear geometry, the large light pole is not visible in the right imagery. Visible is only its shadow, but not enough for a clear identification.
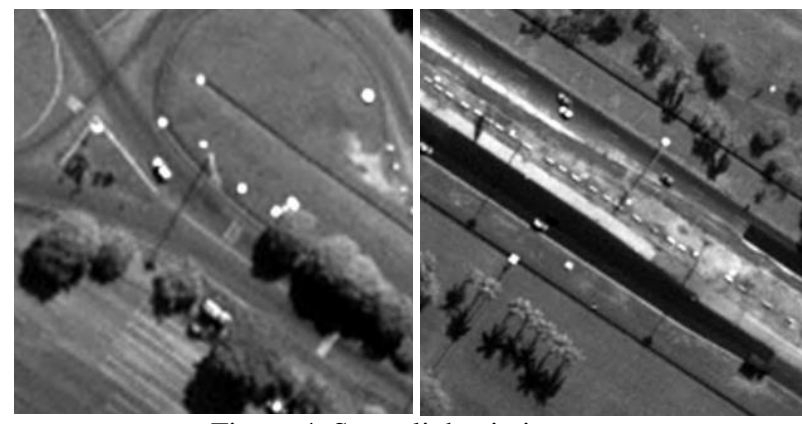

Figure 4. Street lights in imagery

More examples of visibility in optical scenes are not shown in this paper. However, the reader should carefully consider this important aspect.

\subsection{TerraSAR-X enhanced acquisition mode}

The research in this paper was done with the initially available High Resolution SpotLight (HS) acquisition mode. In 2013 the Staring SpotLight (ST) acquisition mode was added. The results published under $3 D$ Point Measurement from Space using TerraSAR-X HS and ST Stereo Imagery. The publication states an improvement of $40 \%$ horizontally and $30 \%$ vertically compared to the independent results researched by CompassData. The ST derived results are not researched by CompassData, however these should be mentioned in this paper. The research was conducted by Airbus Space and Defence with the same reference points obtained by GNSS measurements by CompassData and used in this research.

Both Spotlight modes are characterized by an azimuth antenna steering to a rotation center. The Staring SpotLight mode has its rotation center inside the image scene with a larger steering angle range of $\pm 2.2^{\circ}$. Thus improving image resolution and radiometric performance.
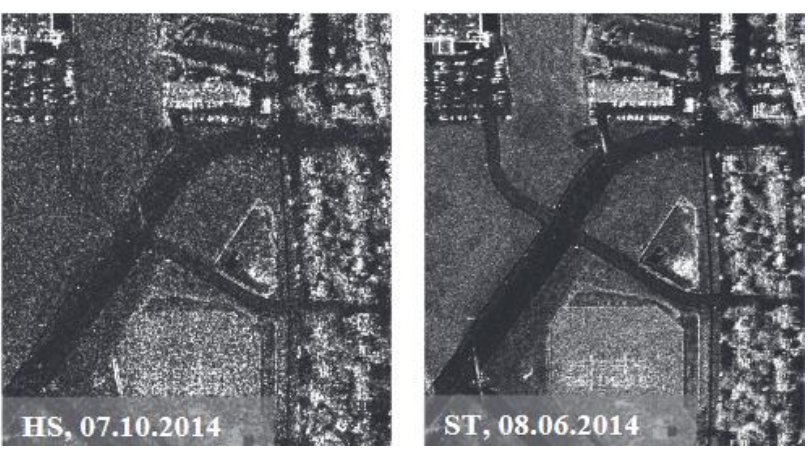

Figure 5. Comparison of a HS scene (left) with a ST scene (right).

The ST scene is less noisy than the HS scene, visible on the asphalt streets and straight lines. This can improve the extraction of non-point features like landscape corners and street intersections.

\section{COMPREHENSIVE TEST STRATEGY}

The primary goal of the test conducted by CompassData, in collaboration with Airbus Space and Defence, was to simulate the workflow in determining RSGCPs $®$ for an inaccessible area anywhere in the world, focusing on standardized man-made features that are found in most parts of the world. For this test, steel towers for electric lines, street-lights, surface contrasts, and traffic islands were examined. Single tests were made with a boulder, a shipping-container, and an electric cabinet.

For validation, Airbus Space and Defence generated coordinates and CompassData surveyed the features with GNSS ground measurements for a comparison. Then the validation was carried out as a blind study.

In addition to regular features, the testing included 'ideal' targets consisting of aluminium corner reflectors directed towards the satellite in each of the passes. This minimized the human error. The separate analysis reveals the system capabilities in a bestcase scenario. The results serve as a reference in regard to the accuracy of the other feature.

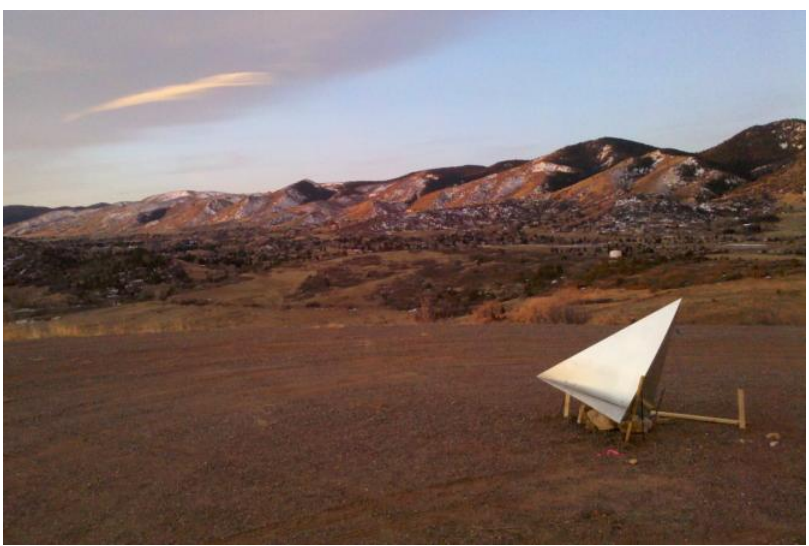

Figure 6. Ideal radar reflector pointed at the satellite

\subsection{Description of the test field}

The test field includes an area of $4 \mathrm{~km}$ by $5 \mathrm{~km}$ close to Denver, Colorado. It sits in the foothills of the Rocky Mountains, located within the Morrison Quadrangle, a site well known to photogrammetrists and remote sensing specialists. 


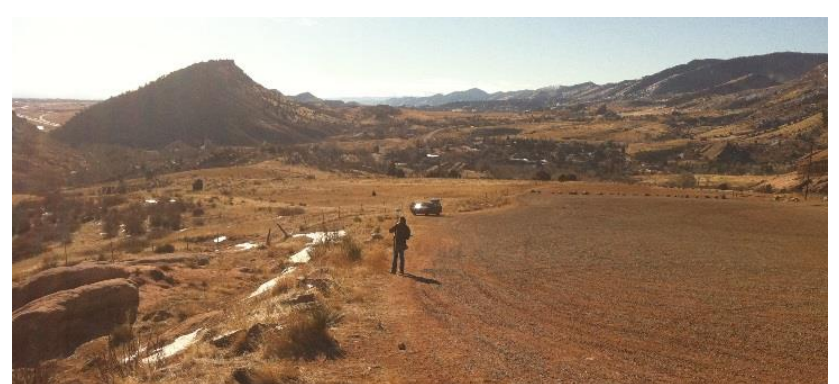

Figure 7. Dynamic topography within the test field

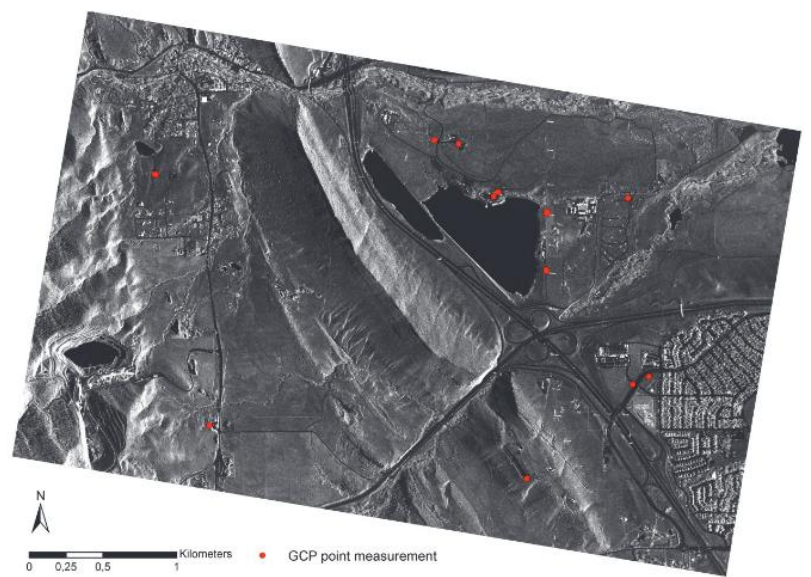

Figure 8. TerraSAR-X dataset and GCPs surveyed on the ground with GNSS.

This test field offers an abundance of different manmade objects embedded in a very dynamic and diverse topography. From east to west, rolling hills of high-plains prairie run up against the first ridge of the Rocky Mountains, which quickly gains 200 meters in a steep incline, in a series of rocky ridges known as the hogbacks and, slightly further west, as the Red Rock formations. These formations consist of solid rock slabs and outcrops several meters high.

The test proved that this extreme mix of variable terrain had no impact on the quality of the RSGCPs. Objects at different elevations were tested and the results showed a consistency regardless of elevation.

\section{RESULTS}

\subsection{TerraSAR-X data}

Acquisition plan for 4 flights using high resolution spotlight scenes.

\begin{tabular}{|l|l|l|l|l|}
\hline Date & Time & $\begin{array}{l}\text { Inclination } \\
\text { Angle }\end{array}$ & $\begin{array}{l}\text { Pass } \\
\text { Direction }\end{array}$ & Mode \\
\hline $2 / 19 / 2011$ & $1: 00: 22$ & 48.75 & Ascending & HS \\
\hline $2 / 22 / 2011$ & $13: 14: 08$ & 26.96 & Descending & HS \\
\hline $2 / 25 / 2011$ & $0: 51: 49$ & 35.76 & Ascending & HS \\
\hline $2 / 28 / 2011$ & $13: 05: 35$ & 42.41 & Descending & HS \\
\hline
\end{tabular}

Figure 9. Acquisition plan for satellite passes

The first set of tests consisted of a comparison between GPS measurements and coordinates of 'perfect' corner-reflectors. Two different kinds of reflectors were placed in the test area.

\subsection{Results for corner reflectors}

Four large aluminium reflectors were constructed out of three triangular shaped planes $(1 \mathrm{~m}$ by $1 \mathrm{~m}$ by $1.5 \mathrm{~m})$ and 3 smaller metal reflectors were made out of three rectangular planes $(0.3 \mathrm{~m}$ by $0.3 \mathrm{~m}$ ).

\begin{tabular}{|l|l|l|l|l|}
\hline $\begin{array}{l}\text { Object } \\
\text { description }\end{array}$ & $\mathbf{H}_{\mathbf{M a x}}$ & $\mathbf{V}_{\mathbf{M a x}}$ & $\mathbf{H}_{\text {Mean }}$ & $\mathbf{V}_{\text {Mean }}$ \\
\hline $\begin{array}{l}\text { 4 large aluminium } \\
\text { reflectors }\end{array}$ & 0.53 & -0.46 & 0.39 & -0.46 \\
\hline $\begin{array}{l}3 \text { small metal } \\
\text { reflectors }\end{array}$ & 0.54 & -0.62 & 0.42 & -0.55 \\
\hline
\end{tabular}

Figure 10. Residuals for 4 large and 3 small reflectors

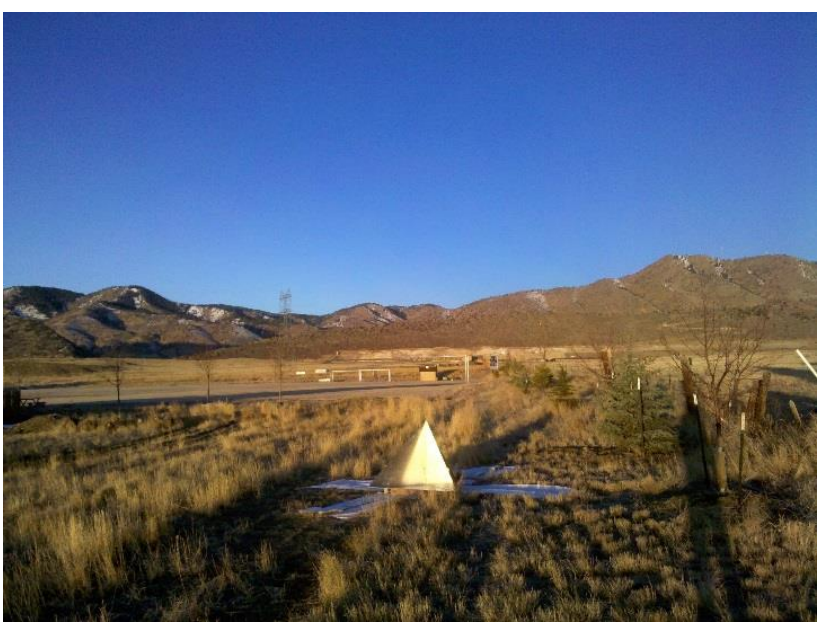

Figure 11 . Large reflector ( $1 \mathrm{~m}$ by $1 \mathrm{~m}$ by $1.4 \mathrm{~m})$

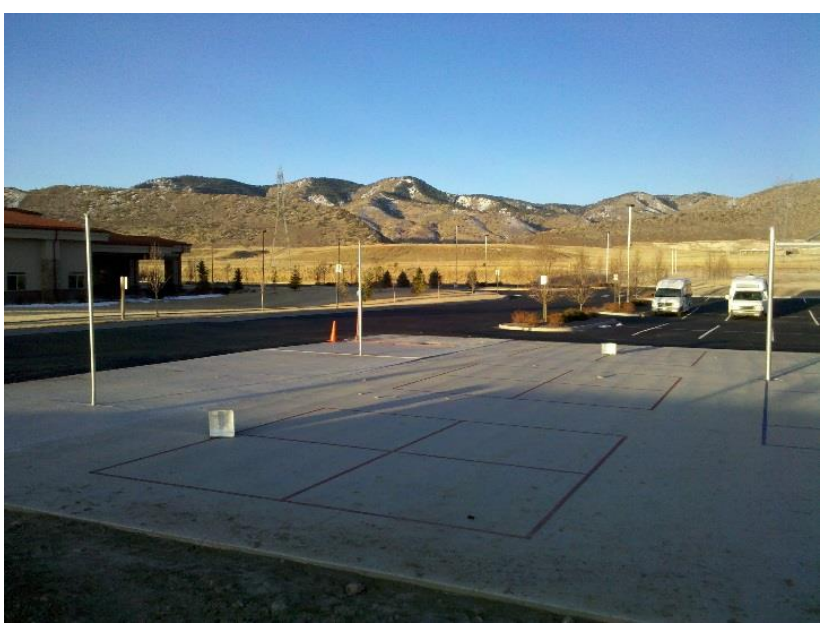

Figure 12 . Small reflector $(0.3 \mathrm{~m}$ by $0.3 \mathrm{~m}$ by $0.4 \mathrm{~m})$ 


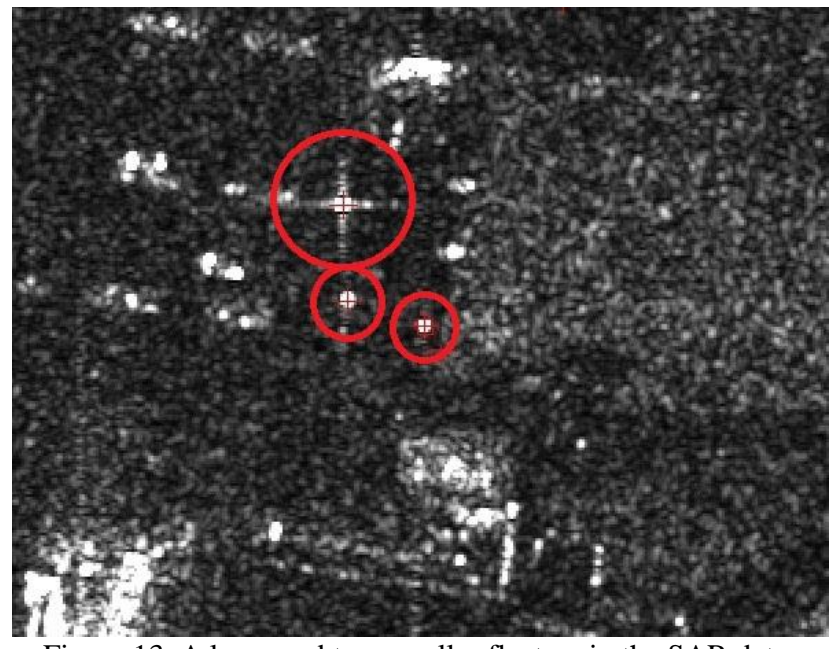

Figure 13. A large and two small reflectors in the SAR data

\subsection{Summarized results for corner reflectors}

The RMS equals $0.41 \mathrm{~m}$ horizontal and $0.50 \mathrm{~m}$ vertical.

The results show a systematic shift. The systematic shift in horizontal position is about $0.3 \mathrm{~m}$ to the West and a vertical lift of $0.5 \mathrm{~m}$ (see scatter plot on next page).

The relative precision between the reflectors is very high. The relative distances determined by satellite, compared to the GNSS measurements, vary by only $0.2 \mathrm{~m}$.

The smaller corner-reflectors ( $0.3 \mathrm{~m}$ side-length) were almost as good when detected by the satellite as the significantly larger reflectors $(1.0 \mathrm{~m})$.

\subsection{Results for remotely sensed features}

For the results of the second 'real world' test, typical RSGCPs®, feature commodities found throughout the world were compared to GNSS measurements with centimetre accuracy. The results show a dependency on the choice of objects, which in a 'real world' scenario might be limited to the availability of objects.

\begin{tabular}{|l|l|l|l|l|}
\hline Features & $\mathbf{H}_{\mathbf{M a x}}$ & $\mathbf{V}_{\text {Max }}$ & $\mathbf{H}_{\text {Mean }}$ & $\mathbf{V}_{\text {Mean }}$ \\
\hline $\begin{array}{l}\text { 5 Steel light- } \\
\text { poles }\end{array}$ & 0.82 & -0.59 & 0.56 & -0.37 \\
\hline $\begin{array}{l}\text { 5 Electric tower- } \\
\text { leg }\end{array}$ & 2.04 & -0.88 & 1.22 & -0.50 \\
\hline $\begin{array}{l}\text { 6 Finished } \\
\text { surfaces * }\end{array}$ & 2.01 & -0.65 & 1.11 & -0.46 \\
\hline 2 Traffic islands & 1.22 & -0.60 & 0.93 & -0.36 \\
\hline 1 Boulder & 1.31 & -1.00 & - & - \\
\hline $\begin{array}{l}\text { 1 Container } \\
\text { corner }\end{array}$ & 2.00 & -0.33 & - & - \\
\hline
\end{tabular}

Figure 14. Results for different features

* Summarized under Finished surfaces are 3 point-features created by asphalt and/or concrete against grass, 2 point-features of asphalt against soil, 1 point-feature created by landscaping with fist-sized rocks against grass.
As shown by the scatter plots, a small systematic shift of approximately $0.3 \mathrm{~m}$ horizontally and $0.4 \mathrm{~m}$ vertically was found in this study.

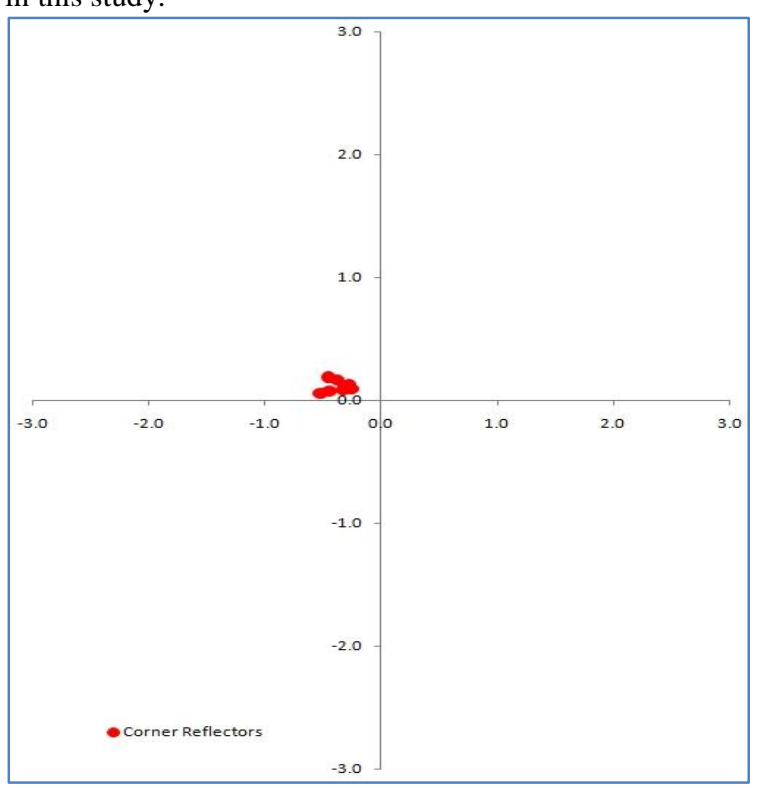

Figure 15. Scatterplot for corner reflectors [Meters]

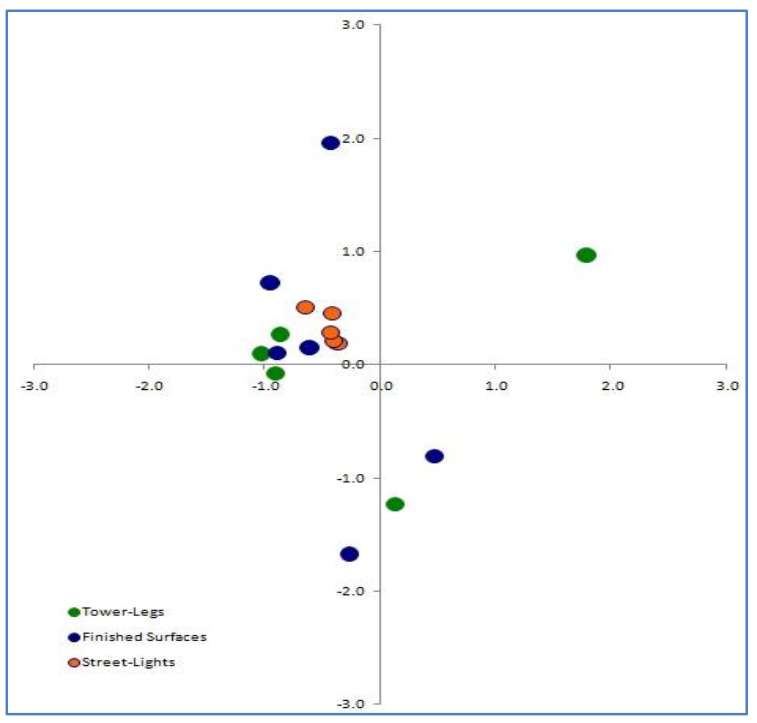

Figure 16. Scatterplot for features [Meters]

The steel light-poles had the best results and the five test features are free of outlines.

Positional outliers can occur, but by a careful selection of features under consideration of their reflectivity and their statistical information, the outliners can be reduced. 


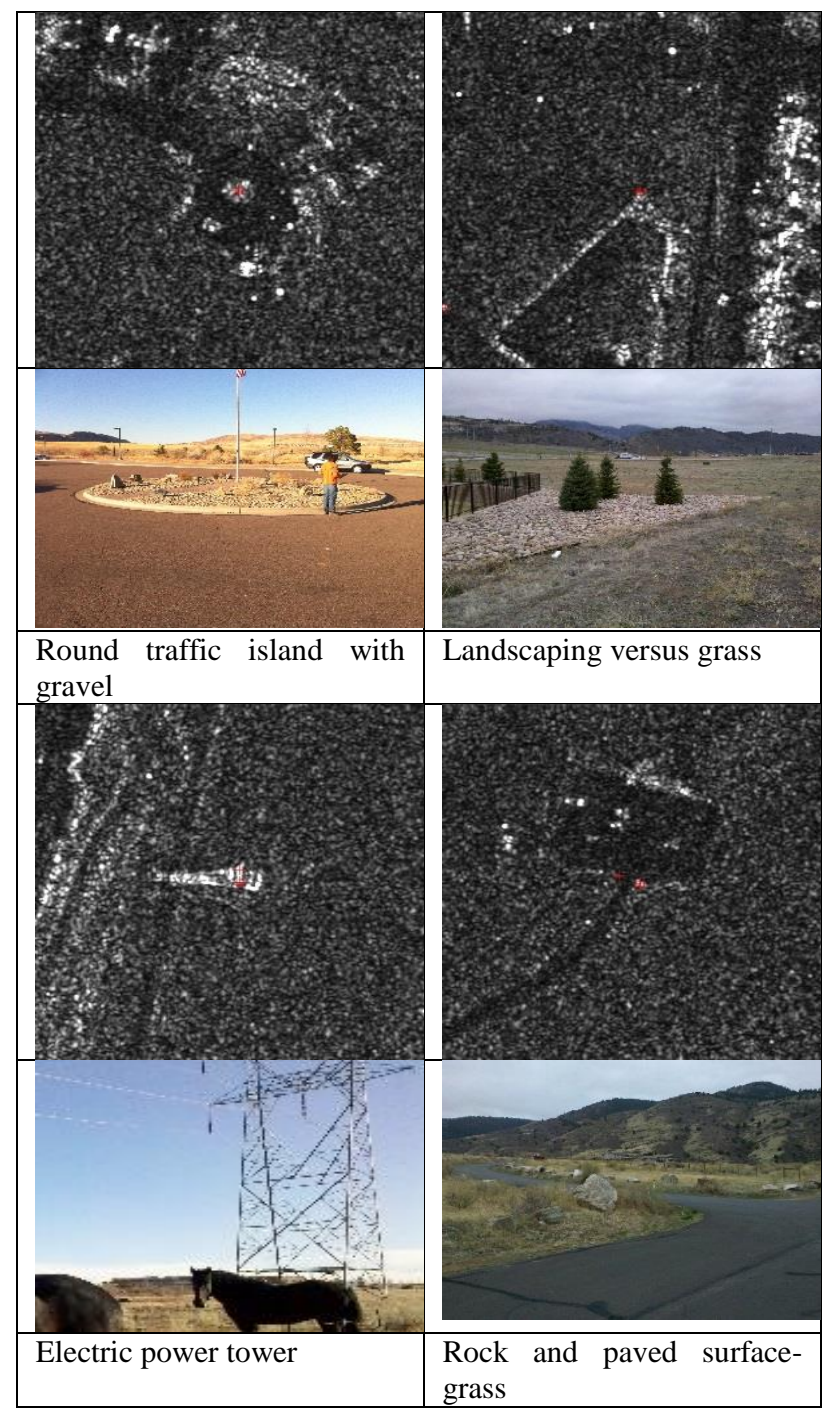

Figure 17. Sample features in SAR data and in the field

The right choice of feature provides the optimal result, as mentioned above the right choice is a combination of visibility of one and the same features in the optical image scene and the TerraSar-X dataset.

The research showed that the right choice for a feature is the key for an optimal RSGCP® result. The feature must fulfil two different requirements:

- $\quad$ First, the feature has to be identifiable in the multispectral or panchromatic scene of an optical sensor.

- Second, the feature must be identifiable in the radiometric imagery.

Therefore, the feature must possess a distinct reflectivity within its surrounding. Two optimal, as well very different, features meeting such requirements are vertical metal poles (i.e., streetlights) and the contrast between smooth and rough surfaces (i.e., traffic islands, corners of an asphalt parking lot surrounded by either grass, gravel, or dirt).

Our results show elevations have a very constant accuracy without any outlier. The effort on the horizontal component has to be greater, especially for a flat feature defined by a change in coarseness. Loose gravel or dirt usually collects on the edges and borders along finished surfaces due to wind and rain. A minor amount could impact the radiometric image, but might not even be visible in the optical image, causing an unwanted offset. Vertical standing metal poles, if available and if visible in the optical image, are preferable.

\section{CONCLUSIONS}

Airbus Space and Defence can produce RSGCPs ${ }^{\circledR}$ from TerraSAR-X with an unrivalled accuracy for a remotely sensed ground truth. Depending on manmade objects, like street lights, power-poles, or cell-antennas in the area of interest, CompassData can provide coordinates with accuracy better than $1 \mathrm{~m}$ (RMSE) in each of the cardinal directions. This quality was independently confirmed in with a comprehensive test strategy and only the High Resolution SpotLight acquisition mode.

The improved Staring SpotLight mode enhances the accuracy and confidence of the extracted point coordinates. The limitations are dependent on image resolution and radiometric performance and the general availability, within the area of interest, of manmade features and their size and quality and how these are displayed in the optical image that is to be georeferenced.

Traditional terrestrial based survey is still the ideal source for control but this study has confirmed the usability of RSGCPs $®$ in inaccessible areas or where access is denied.

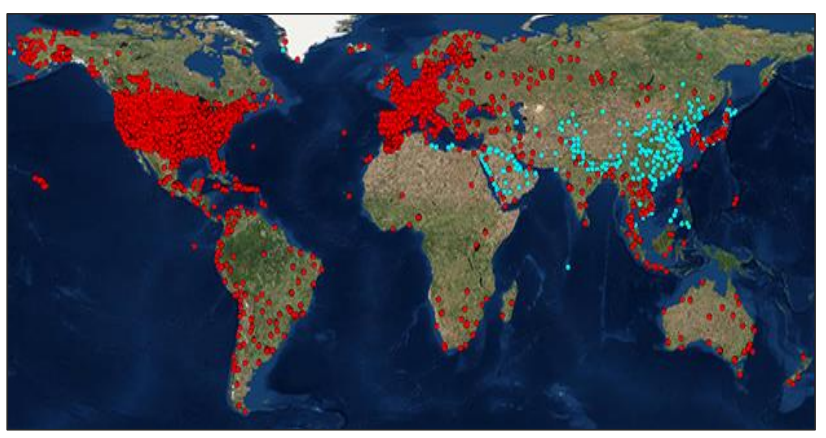

Figure 18. Archived RSGCPs ${ }^{\circledR}$ (blue) supplementing the GCPs (red) in CompassData's global archive

\section{ACKNOWLEDGEMENTS}

Thanks to Laura Henrichs at Airbus Space and Defense for the supporting material.

The figures $1,2,5,9$, and partially 12 are courtesy of Airbus Defense \& Space.

RSGCP $®$ is a registered Trade Mark of CompassData, Inc.

\section{REFERENCES}

Nonaka, T., Evaluation of the Geometric Accuracu of TerraSAR-X, ISPRS, Vol. XXXVII, Part B7, Beijing 2008, pp $135-140$

Mittermayer, J,., Wollstadt, S. Prats, P. Schreiber, R., Koppe, W., Staring Spotlight Imaging with TerraSAR-X. IEEE International Geoscience and Remote Sensing Symposium (IGARSS), 2012 IEEE International, Munich, Germany, pp. 1606-1609 
Koppe, W., Hennig, S., Henrichs, L., 3D Point Measurement from Space using TerraSAR-X HS and ST Stereo Imagery, DGPF Tagungsband 24 /2015, Hamburg, Germany, ISSN 09422870 ,

Koppe, W., Wenzel, R., Hennig, S., Janoth, J., Hummel, P., Raggam, H., Quality Assessment of TerraSAR-X derived Ground Control Points, 2012, Geoscience and Remote Sensing
Symposium (IGARSS), 2012 IEEE International, Munich, Germany, pp. 3580-3583

Raggam, H., Perko, R. Gutjahr, K., Kiefl, N., Koppe, W., Hennig, S., Accuracy Assessment of 3D point Retrievel from TerraSar-X, EUSAR $20108^{\text {th }}$ European Conference on Synthetic Aperture Radar, Aachen, Germany, pp. 1-4 